Chapter 2

\title{
Preoperative Planning of Total Knee Replacement
}

\author{
A.O. Erdogan, N.S. Gokay and A. Gokce \\ Additional information is available at the end of the chapter \\ http://dx.doi.org/10.5772/55023
}

\section{Introduction}

Total knee replacement (TKR) surgery is the gold standard method in the treatment of end stage knee arthritis with a high success. Relieving pain effectively, restoring range of motion and improving function are the major goals of arthroplasy. Patient satisfaction rates were reported as 90\% to 95\% after TKR. [1-3] Better clinical results were gained in younger population than the older patients. Unfortunately, primary osteoarthritis is seen mainly in the elderly. However, inflamatory arthritis, trauma, avascular necrosis and hemophilia are some of the secondary causes leading osteoarthritis in younger population.. Nearly 1 million TKR surgeries are performed on earth per year and this number is increasing day by day, with the novel designed surface technologies. Despite the fact that new implant technologies secure better results, a revision procedure may be required in some of the patients. Ofcourse the amount of revision surgeries is also increasing in parallel with primary TKR surgeries. Unfortunately, the clinical result of revision total knee arthroplasty isn't succesfull enough as primary total knee arthroplasty.

Polyethylene (PE) wear, aseptic loosening, instability and infections are counted among the factors leading revision surgery $[4,5]$. The incidence of PE wear is decreased nowadays by the development of ultra high cross-linked polyethilens (UHMWPE). PE wear is a serious problem leading to aseptic loosening. Malalingment, malrotation and soft tissue imbalance after TKR surgery are the other factors which may cause in aseptic loosening. Instability and problems related with patella are among the other situations in which revision surgery may be requiredIt is very clear that surgical technique of primary TKR is the main risk factor for early failures [6, 7]. Extensive preoperative planning is very important considering aforementioned particulars to achieve accurate prosthetic seating with proper axial alignment. In this chapter, the preoperative planning issues including pre-operative preparations of patient and surgeon will be discussed. 


\section{Pre-operative clinical evaluation}

Pre-operative clinical evaluation is very substantial in assessing the current medical status and determining the relative risk profile of the patient. It is very important for the orthopaedic surgeon to understand the basic principles and nuances involved. Preoperative planning initiates with selection of an apropriate candidate for TKR procedure. The patient's expectations and general risk factors plays an import role in this decision. Questioning of the patient's complaints and life quality, assesment of the medical condition and detailed physical examination are the essentials of preoperative clinical evaluation.

\section{Indication of TKR}

Total knee replacement was found to be quite effective in terms of improvement in healthrelated quality of life dimensions, with the occasional exception of the social dimension. The underlying disease is not a life threatening one. The primary indications for total knee arthroplasty are pain and loss of function. Clinical evaluation of the patient has to be initiated by questioning of the complaints and disabilities.

There must be a significant and disabling pain which is resistant to the medical treatment. Spinal disease, ipsilateral hip pathologies, peripheral vascular disease, meniscal pathologies, and bursitis of the knee should be considered in differential diagnosis. The real source of the knee pain must be displayed.

The patient's quality of life should be questioned and significant reduction might be taken into consideration It should be remembered that the patients, which are less satisfied following TKR, are the ones with mild pre-operative complaints. All conservative treatment modalities should be exhausted before surgery. The frequent indications for TKR surgery are given on table 1.

\begin{tabular}{l}
\hline Osteoarthritis \\
Inflammatory arthritis \\
Rheumatoid arthritis \\
Osteochondromatosis \\
Villonodular synovitis \\
Metabolic arthritis \\
Osteonecrosis \\
Gout, pseudogout \\
Posttraumatic arthritis \\
Intraarticular fractures \\
Malaligned ankylosis \\
Failed high tibial osteotomies
\end{tabular}

Table 1. The major indications for total knee arthroplasty 


\section{Contrendications of TKR}

Contraindications must be well known and considered before the surgery decision. Absolute contraindications for total knee arthroplasty include knee sepsis, chronic infection, extansor mechanism dysfunction, severe vascular disease, recurvatum deformity secondary to muscular weakness, presence of well functioning knee arthrodesis. There are also relative and contraversial contraindications. These include medical conditions that preclude safe anesthesia, inadequate soft tissue coverage, morbid obesity, neuropathic arthropathy, and history of osteomyelitis around knee joint. A preoperative condition that can adversly affect the patient's outcome can be considered as a relative contraindication.

\section{Medical condition of the patient}

A comprehensive medical history and physical examination are the cornerstones of the preoperative assessment. Patient's medical comorbidities should be optimized preoperatively. Cardiovascular diseases, cerebrovascular diseases, pulmonary diseases, neurologic conditions, rheumatoid arthritis, uropathy, morbid obesity, haematologic and endocrine diseases are the important situations which may affect the intraoperative and postoperative morbidity and mortality.. Drug history of the patient must be questioned too. Sources of infection such as dental, dermatological, urological or respiratory tract infections must be ruled out. Age alone was not found to be an obstacle for an effective surgery, however age related comorbidities should be considered. Additional risks for infection, previous surgeries should be adressed before surgery. Every patient must have a general medical evaluation including laboratory tests, electrocardiogram and chest graphy. The type of the procedure also may enhance the medical risks. For example, simultaneously performed bilateral TKR may be associated with an increased risk of cardiac, respiratory or neurological complications. Low preoperative mental health has also an influence on outcome after TKR [10].

The detailed physical examination should include the range of motion, ligament status, circulation and skin condition evaluation. Furthermore alignment of lower extremities, patellar instability and gait analysis should to be assessed. Additional knowledge and scientific dissemination of surgery outcomes should help to ensure better management of the patients after total knee arthroplasty and to optimize the procedure [12].

Underlying or predisposing orthopaedic diseases, which may interact the outcome of TKR should be reviewed previously. Examples of such common musculoskeletal conditions are as follows: 1) Nerve entrapments affecting motor functions of lower extremity, 2) Adjacent joint degenerative arthritis 3) Presence of any inflamatory arthritis, which may flare after surgery 4) Any musculoskletal disability affecting postoperative mobility 5) Thromboembolic history or predispositions. 


\section{Radiographic assesment}

An apropriate musculoskeletal radiologic study is essential in planning of TKR. The presence of bone defects might be detected and pre-operative decision whether to use a primary or revision implant should be given. Determination of the joint line is also important in determining the flexion/extension space and balance of the ligaments.

Tracking of the quadriceps mechanism and rotational deformites are other important issues for patellar stability, however may not be assessed on direct radiographs especially in deformed knees with fixed valgus position.

Conventional radiographs are usually adequate for initial radiographic evaluation to confirm the diagnosis or assess the severity of the disease. Preoperative radiographic planning for total knee arthroplasty begins by obtaining a high quality standing antero-posterior (AP) and lateral 52-inch casette graphies. Additional views such as Merchant, tunnel, patella sunrise, orthoroentgenogram or advanced imaging modalities such as CT, MRI may be necessary in extraordinary conditions such as congenital dislocation of the patella, post traumatic deformities, severe deformities, tumors and congenital anomalies.

\subsection{AP view}

The AP view should be obtained with the patient in a standing position. The joint space on the weight bearing AP film should be more than $3 \mathrm{~mm}$ or within $50 \%$ of the joint space of the contralateral knee. Normally, the lateral joint space is wider than the medial space. Articular surfaces of the medial and lateral joint compartments may be best assessed on this view. The presence of associated osteophytes and subcondral bone changes are some of the findings which may be seen on AP graphies.

\subsection{Lateral view}

The lateral aspect of the knee is leaned against the $x$ ray film cassette and flexed $30^{\circ}$. The excessive glide in flexion may be an indicator of posterior cruciate ligament tightness and therefore PCL sacrifiying prosthesis must be considered before the surgery. Patellar height should be assessed on this view using Insall-Salvati ratio. The Insall-Salvati is the ratio of the patellar tendon length (LT) to the length of the patella (LP) (Figure 1). The values above 1,2 is considered as "Patella Alta", while the values belove 0,8 is considered as "Patella Infera". Suprapatellar and posterior regions must be evaluated in terms of detecting the loose bodies.

Posterior of the knee joint is easily observed on this view and overhanging bone structure or osteophytes should be determined. If left unresected, this overhanging bone can impinge on tibial PE and result with flexion limitation and early wear of the PE.

The lateral view may also allow size selection of the femoral component (Figure 2). Oversizing will create tightness in fexion and increased tension in quadriceps mechanism and undersizing will create looseness in flexion and anterior cortical notching of the femur is also inevitable. Careful preoperative planning with using templates aims to achieve the correct AP dimension for restoring normal kinematics and quadriceps muscle function. 


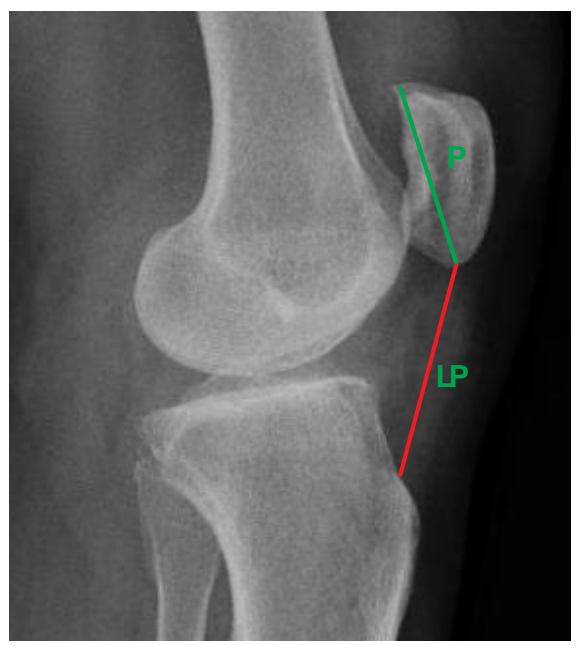

Figure 1. Patella height is assessed via measuring Insall-Salvati ratio (LP/P) on lateral graphies

\subsection{Standing orthorontgenogram (52-inch casette three joint view)}

Standing ap view of the lower extremities from hips to ankles is helpful preoperatively for assessing overall alignment (mechanical axis) of the lower extremity. The knee is normally in 7 degree of valgus alignment on AP view.

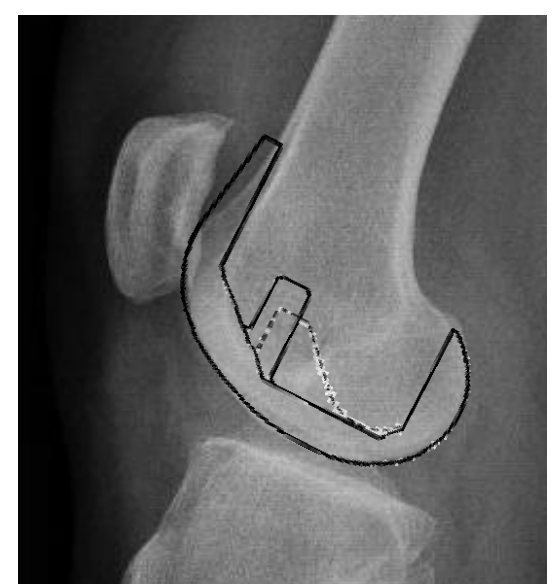

Figure 2. Proper size of the femoral component is assessed via evaluation of the pre-operative lateral radiographs.

The orthorontgenogram allows the surgeon to determine the varus or valgus alignment of the knees, leg length discrepancy and the presence of extraarticular deformities. 
It is essential to ensure to take this radiograph in neutral rotation of the legs to obtain the most accurate measurements. It should be confirmed via inspecting the relationship between the tibia and the fibula, distally and proximally.

\subsection{Merchant view}

Merchant view helps to assess the patellofemoral alignment, trochlear grove and articular surfaces. Preoperatively patellar subluxation seen on this view should alert the surgeon for lateral release of the patella during TKR. Merchant view demonstrates both the patellar and trochlear surfaces. Subluxation can be assessed by measuring the congruence angle (Figure 3). Congruence angle demonstrates the relationship of apex of patella with the trochlear grove's bisector. Two lines are drawn to measure the congruence angle. First line is the bisector line of femoral sulcus angle and it establishes a zero reference line. Second line is drawn from the apex of the sulcus angle to the lowest point of the patellar articular ridge.

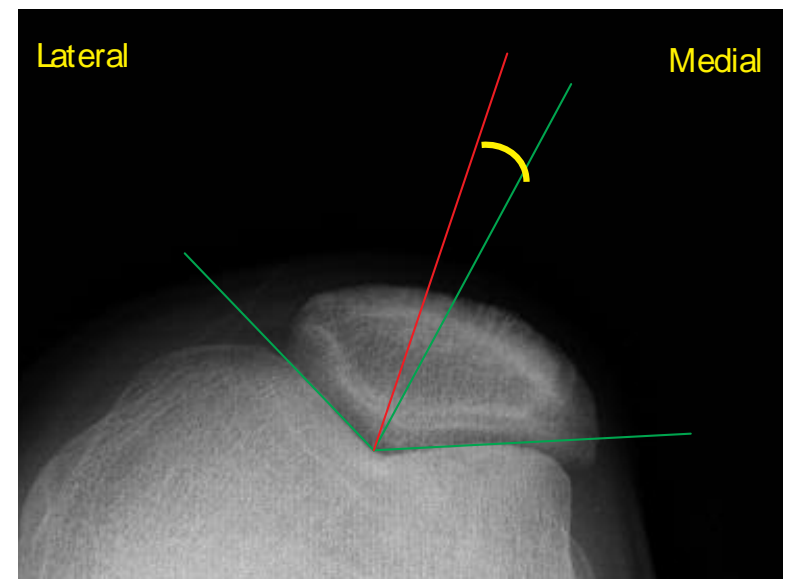

Figure 3. The congruence angle (a) lower than $16^{\circ}$ to lateral or medial direction is considered as normal.

If congruence angle is lateral to the reference line this means the angle is positive while angles medial to the reference lines are considered as negative. The angles lower than 16 degree are considered as normal.

\subsection{Tunnel view}

Tunnel view shows the posterior aspect of the intercondylar notch and it is useful to evaluate osteocondritis dissecans or intraarticular loose bodies in young patients.

\subsection{Patella sunrise view}

This is called the sunrise view because the patella appears to be rising over the horizon. The sunrise view also helps to identify the patellar malalignment. 


\section{Surgeon's preperation}

Preoperative planning is essential component for surgeon in TKR to obtain desired clinical outcome and radiographic result. Correct implant selection with proper size is one of the important stage in preoperative planning. Patient's and surgeon's expectations should be taken into consideration pre-operatively to improve the outcomes and increase the satisfaction after operation [12]. Pre-operative planning of the bone cuts may simplify the surgery. Positioning of the components is very important in acquiring the accurate alignment. Mechanical axis measurement and alignment evaluation on AP and lateral planes may performed on standing X-rays. Also entry points for intramedullary guide pins should be determined for the correct component position.

During the process of planning of TKR, the surgeon aims to obtain the optimal fitting of the implant and the optimal extremity alignment. This is possible for the surgeon by thinking three-dimensionally. The prediction of the surgery pre-operatively should improve the precision of the process and shorten the length of the operation. It should also reduce the complication rate.

\section{Templating}

Most of the knee replacement systems provide a large number of options for treating conditions that are encountered during surgery. The different systems serve the surgeons different sizes of implants in a wide range. Intra-operative determination of the implant sizes was found more accurate than the pre-operative templating. However pre-operative templating is useful in obtaining proper implant positioning. Working with templates is widely accepted approach, which can help determine component sizes, predict amounts of bony resection, and anticipate surgical steps. Acetate templating was found accurate and reliabile [13]. However, with widespread use of digital radiographs the use of digital templating has recently grown due to advances in reducing errors associated with manipulating films and templates (Figure 4).

Combining digital templating with picture archiving and communication system PACS brings advantages include saving health care costs, quick and reproducible planning, remote access, and the ability to keep a permanent digital record of the surgical plan.

Templating should also work in preparing the surgeon to some extreme scenarios which may be encountered during surgery. For example; Standart cruciate retaining or sacrifiying implant systems are usually enough to solve the problem in large majority of cases. Constrained or even more complicated sytems with extension stems or wedges may be necessiated in small number of primary cases (Figure 5). Modular systems should be required due to intraoperative complications such as fractures or ligament tears.

Constrained condylar knee prosthesis (CCK) is commonly used in total knee replacement to overcome the severe ligamentous imbalance problem. CCK may also be preferred if the lateral 


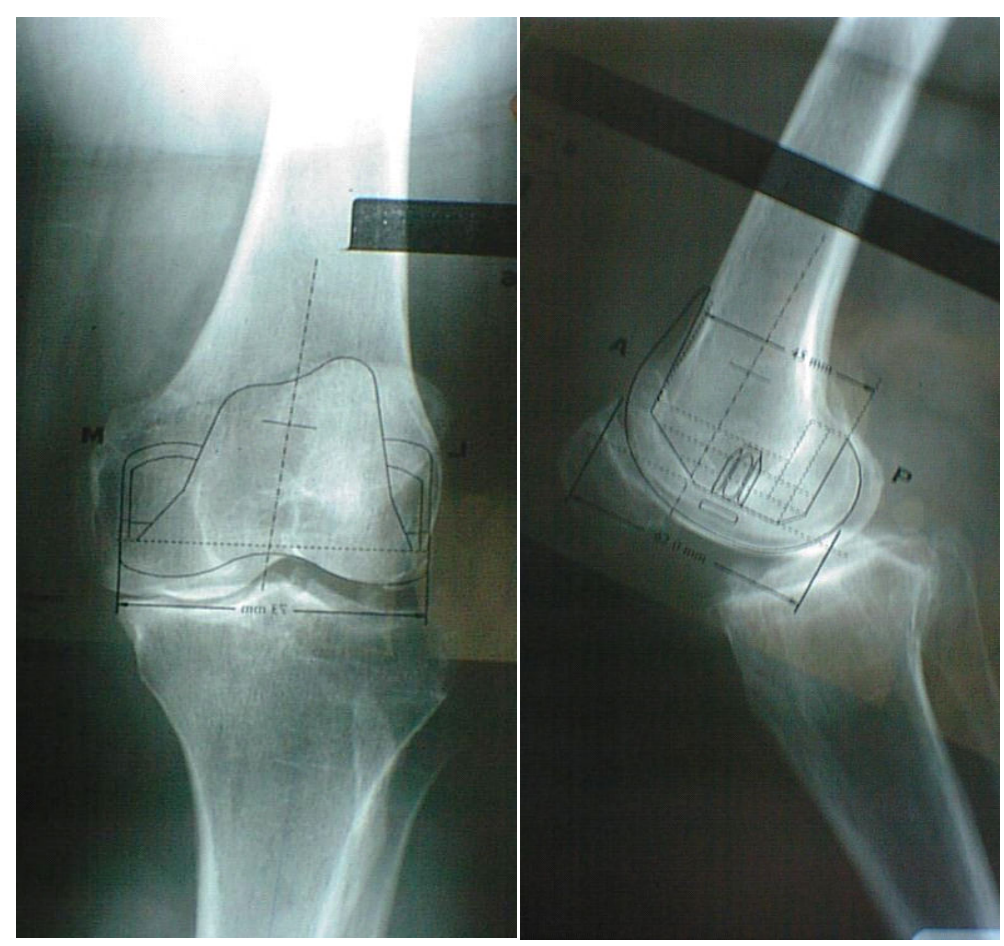

Figure 4. Templating

collateral ligament laxity more than $2 \mathrm{~mm}$ persists, even after adequate medial soft tissue release in patients with varus deformity of greater than $20^{\circ}$.

Although women have greater functional limitations than men, at the time of the surgery, they recover faster in early post-operative period. Women also have a greater improvement in WOMAC scores than men after primary surgery. Specific anatomic differences are blamed to be responsible for gender-specific differences after TKR. However, there are conflicting data in the literature regarding these gender-specific outcomes [14].

There are also efforts in reducing costs and improving quality of care, which may planned in preoperative period. Template-directed instrumentation was proposed for simplifying the surgical instruments and saving sterilization costs of unnecessary implant trays as saving measurement. In this novel approach, surgical planning combines digital templating with prepared instruments and components used intraoperatively [15].

Computer based navigation should be prefered in complex cases to overcome the heavily distorded anatomy or malalignment. There are also several quite recent technologies, like patient specific surgical cutting guide systems or robotic assisted implantations in cases of surgeon's special interest. Beside the medical aspects surgeons have to deal with cost considerations and even discuss with the patient in preoperative planning period [16]. 


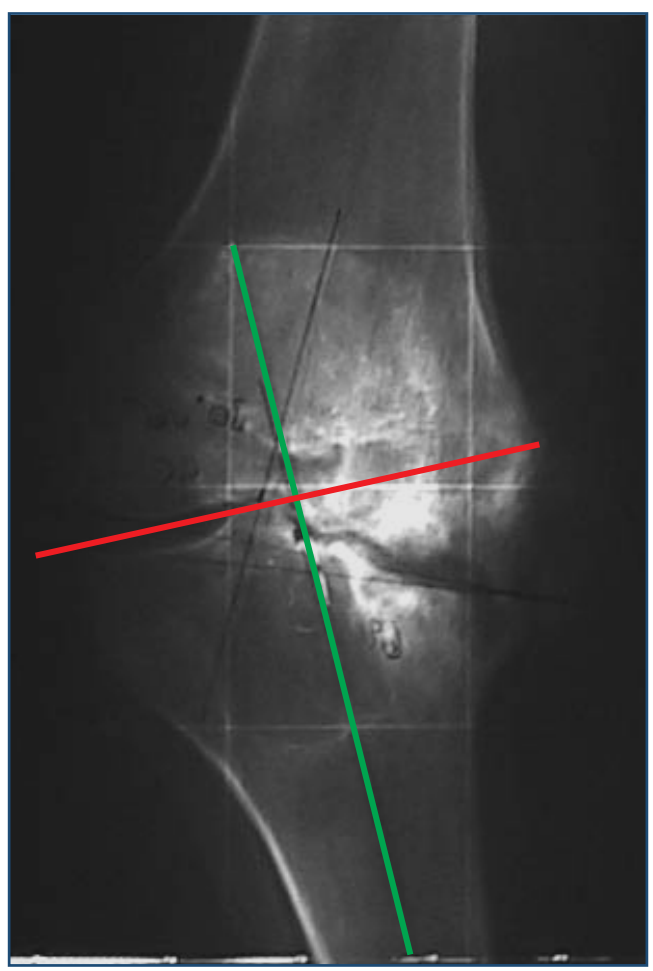

Figure 5. Wedge and modular extension stems should be considered in patients which present with a such pre-operative radiograph.

\section{Author details}

A.O. Erdogan, N.S. Gokay and A. Gokce

Namik Kemal University School of Medicine, Department of Orthopaedics and Traumatology, Tekirdag, Turkey

\section{References}

[1] Colizza, W. A, \& Insall, J. N. Scuderi GR: The posteriorstabilized knee prosthesis: Assessment of polyethylene damage and osteolysis after a ten-year minimum followup. J Bone Joint Surg 77A:(1995). , 1713-1720. 
[2] Emmerson, K. P, \& Moran, C. G. Pinder IM: Survivorship analysis of the kinematic stabilizer total knee replacement: A year follow-up. J Bone Joint Surg 78B:441-445, (1996). , 10-14.

[3] Ranawat, C. S, \& Luessenhop, C. P. Rodriquez JA: The press-fit condylar modular total knee system: Four to six year results with a posterior-cruciate-substituting design. J Bone Joint Surg 79A:(1997). , 342-348.

[4] Robertsson, O, \& Lidgren, L. Annual Report- The Swedish Knee Arthroplasty Register. Annual Report SKAR (2008). , 1-37.

[5] Mulhall, K. J, Ghomrawi, H. M, Scully, S, Callaghan, J. J, \& Saleh, K. J. Current etiologies and modes of failure in total knee arthroplasty revision. Clin Orthop Relat Res (2006). , 446, 45-50.

[6] Manley, M, Ong, K, Lau, E, \& Kurtz, S. M. Total Knee Arthroplasty Survivorship in the United States Medicare Population Effect of Hospital and Surgeon Procedure Volume. J Arthroplasty (2008).

[7] Schroer, W. C, Calvert, G. T, Diesfeld, P. J, \& Reedy, M. E. LeMarr AR. Effects of increased surgical volume on total knee arthroplasty complications. J Arthroplasty (2008). Suppl 1):61-67.

[8] Crekarell, J. R, \& Guyton, J. L. Arthroplasty of ankle and knee. Canale ST (ed). Campbell's operative orthopaedics. 10th edition, St Louis: Mosby, (2003).

[9] Aydogdu, S. ve ark. Diz artroplastisinde genel ilkeler endikasyonlar. Ege R. Diz sorunları. Ankara, bizim büro basımevi, (1998). , 439-459.

[10] Vissers, M. M, Bussmann, J. B, Verhaar, J. A, Busschbach, J. J, Bierma-zeinstra, S. M, \& Reijman, M. Psychological factors affecting the outcome of total hip and knee arthroplasty: a systematic review. Semin Arthritis Rheum. (2012). , 41(4), 576-88.

[11] Ethgen, O, Bruyère, O, Richy, F, Dardennes, C, \& Reginster, J. Y. Health-related quality of life in total hip and total knee arthroplasty. A qualitative and systematic review of the literature. J Bone Joint Surg Am. (2004). A(5):963-74., 86.

[12] Ghomrawi, H. M, Mancuso, C. A, Westrich, G. H, \& Marx, R. G. Mushlin AI Discordance in TKR Expectations Between Patients and Surgeons; Expectations Discordance Study Group.Clin Orthop Relat Res. (2012). Jul 21.

[13] Peek, A. C, Bloch, B, \& Auld, J. How useful is templating for total knee replacement component sizing? Knee. (2012). Aug;doi:j.knee.2011.03.010. Epub 2011 May 10., 19(4), 266-9.

[14] Liebs, T. R, Herzberg, W, Roth-kroeger, A. M, Rüther, W, \& Hassenpflug, J. Women recover faster than men after standard knee arthroplasty. Clin Orthop Relat Res. (2011). , 469(10), 2855-65. 
[15] Hsu, A. R, Gross, C. E, Bhatia, S, \& Levine, B. R. Template-directed Instrumentation in Total Knee Arthroplasty: Cost Savings Analysis. Orthopedics. (2012). Nov 1;35(11):edoi:, 1596-600.

[16] S.David Stulberg Chapter 3 Preoperative Planning and Prosthetic Selectin in book Knee Arthroplasty edited Thomas P. Sculco and Ermanno A. Martucci, New York: Springer Wien; 2002. 250. 
